\title{
Reduction glossoplasty using a modified Morgan technique: a technical note
}

\author{
Ahmed Zaroual`, Zakaria Aziz, Allaye Garango, Bredel-Djeri Mabika, Naouar Ibnouelghazi, \\ Nadia Mansouri-Hattab
}

Service de stomatologie et chirurgie Maxillo-faciale et esthétique, CHU Marrakech, Morocco

(Received: 25 February 2016, accepted: 27 December 2016)

Keywords: macroglossia / glossoplasty / phonation / malocclusion

\begin{abstract}
Macroglossia is defined as an increase in volume of the movable part and/or the base of the tongue. This condition is responsible for many functional (phonatory, masticatory, occlusal, orthodontic, and gustatory) and psychological disorders. A well-planned surgical intervention may improve things. We present in this technical note in which the results of a reduction glossoplasty using a modified Morgan technique was performed on three young patients with true congenital macroglossia. The technique is discussed in detail.
\end{abstract}

\section{Introduction}

Macroglossia is defined as a condition in which the tongue protrudes because its volume is too high compared to the oral cavity (Fig. 1). Depending on the underlying cause, macroglossia can be divided into pseudomacroglossia and true macroglossia. Surgical procedure by reduction glossoplasty (by decreasing the length and width of the tongue) aims to improve lingual morphology and subsequent improvement in tongue function.

\section{Technical note}

The procedure was conducted under general anesthesia with nasotracheal intubation, the patient was placed in a dorsal position with the head resting on a headrest. The tongue was then maintained outside the oral cavity by three sutures, maintaining its symmetry, so that the incision traces could be drawn. Excision was performed according to Morgan transfixing technique. The initial full-thickness incision was made in the middle and back of the tongue following an elliptical trace approximately $4 \mathrm{~mm}$ from the taste buds up to the ventral aspect (Fig. 2). This incision was completed by side incisions forming a "keyhole" on the dorsal aspect and a "W" on the ventral aspect (Fig. 3). The purpose of this "W"type incision is to maintain the lingual frenulum, which will be sacrificed with a racket aspect after the partial glossectomy if a " $\mathrm{V}$ "-shaped incision (as described by Morgan) is used

\footnotetext{
*Correspondence: zaroualcmf@gmail.com
}

(Fig. 4). Closure was finally made end to end in three planes after hemostasis was assured (Fig. 5). Postoperative care was simple. The slight edema of the floor of the mouth (without respiratory signs) and mild hypoesthesia of the tip of the tongue resolved over time. The resumption of sensorimotor and gustatory functions was back to normal 10 days after surgery, with preservation of lingual symmetry and good oral capacity. Postoperative medical treatment consisted of analgesia (paracetamol $60 \mathrm{mg} / \mathrm{kg} /$ day for 5 days and then as required), intravenous prophylactic antibiotics for $24 \mathrm{~h}$ and then orally for 7 days total (amoxicillin + clavulanic acid $50 \mathrm{mg} / \mathrm{kg} /$ day), local antisepsis by mouthwash and dietary advice (cold meals for $48 \mathrm{~h}$, then soft food).

Patients are subsequently referred to occlusal and phonatory rehabilitation so as to recreate physiological conditions that prevent the recurrence of functional or morphological disorders.

\section{Discussion}

Macroglossia is a general term used to describe a protruding tongue beyond the teeth during the natural resting phase [1]. This can compromise the integrity of the upper airways because of obstruction and often results in obstructive sleep apnea $[1,2]$. We refer to pseudomacroglossia or relative macroglossia when the lingual protrusion is because of specific anatomical conditions [3]. This is the case with constituent endoalveoli, where the dental/lingual axis reduces the volume available for the tongue. A similar lingual protrusion is found in cases of a low mandible (Down and Pierre-Robin syndromes). 


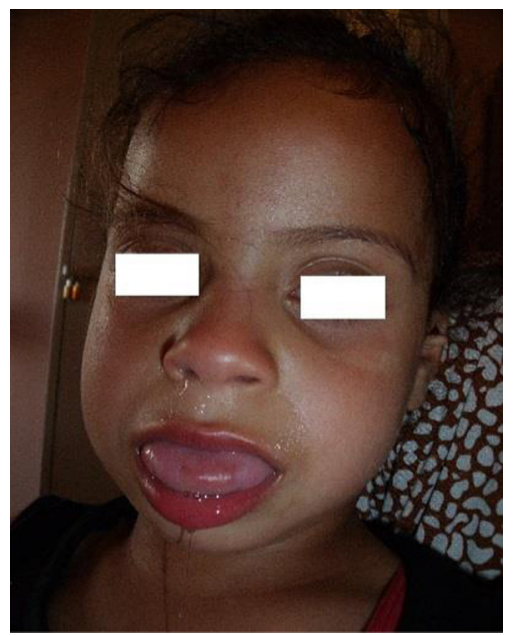

Fig. 1. Clinical aspect of true macroglossia: the tongue is in protrusion because of the fact that its volume is too large compared to the oral cavity.

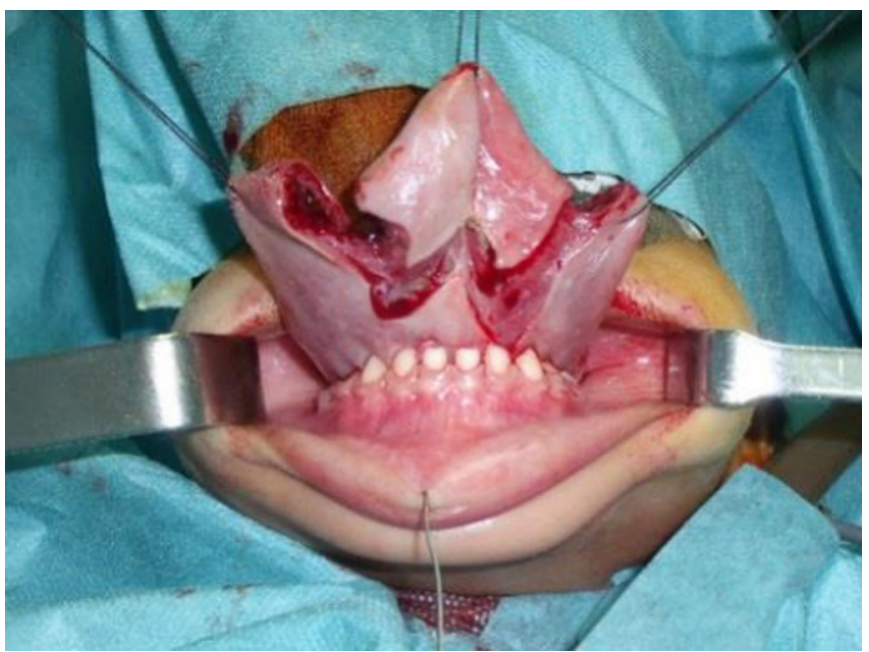

Fig. 3. "W"-shaped incision of the ventral side of the tongue, enabling the preservation of the lingual frenulum.

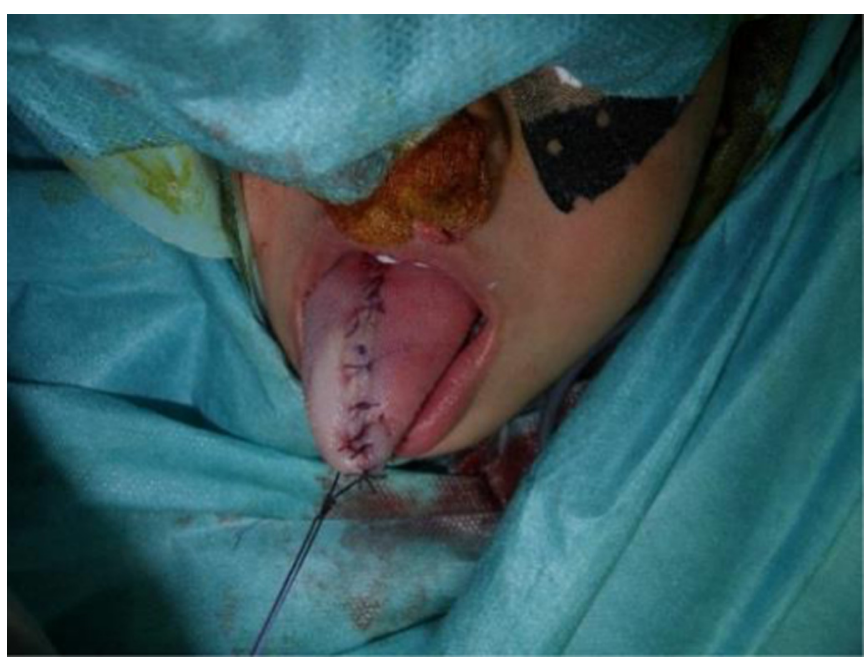

Fig. 5. Images after multilayer closure. The tongue reduction has been achieved in both length and width.

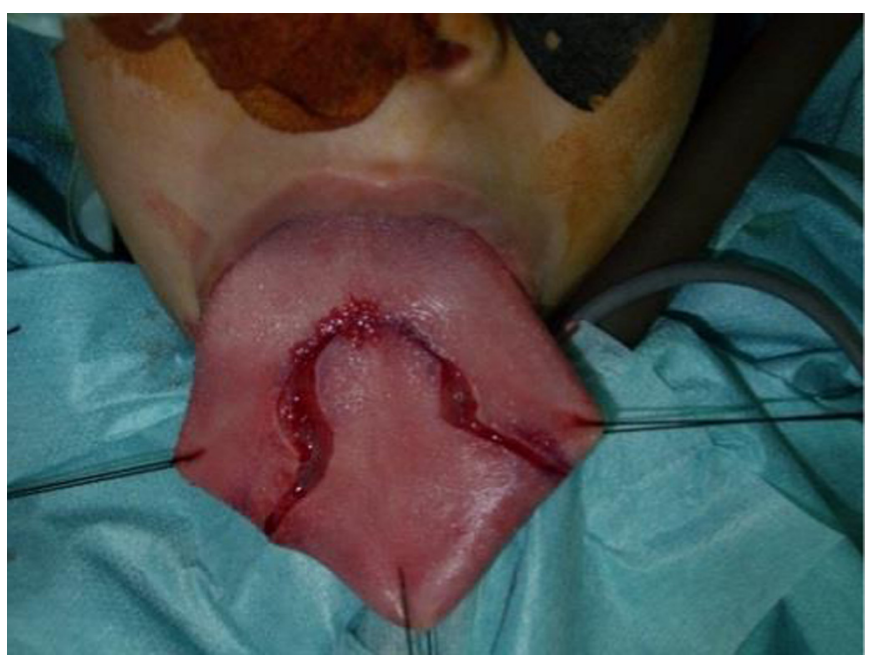

Fig. 2. Dorsal incision of the tongue. It starts with a 4-mm central, elliptical incision of the taste buds and is complemented by two lateral incisions, forming a "keyhole".

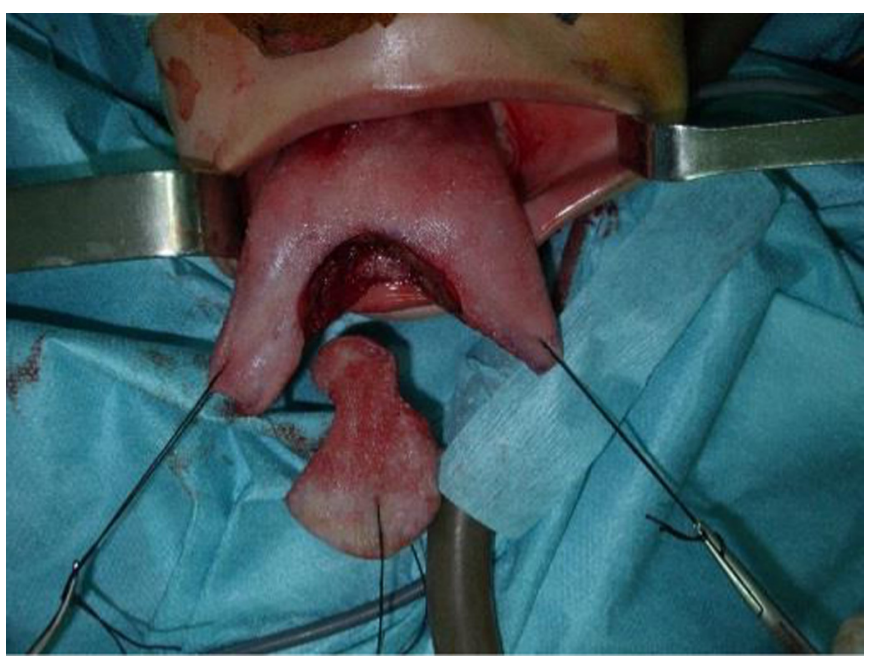

Fig. 4. The excision is transfixing, racket-shaped.

True macroglossia is characterized by hypertrophy with hyperplasia of glottic muscles or abnormal tissue hypertrophy. It could be due to congenital or acquired causes. Congenital factors include muscular hypertrophy, glandular hyperplasia, neonatal diabetes as well as certain genetic diseases, such as trisomy 21 and Beckwith-Wiedemann syndrome. Acromegaly, myxedema, hyperthyroidism, amyloidosis, and tongue tumors are acquired etiologies $[4,5]$.

Reduction glossoplasty surgery is indicated in cases of growth disorders of the dental arch, with a tendency to classIII skeletal patterns and pro-alveoli, phonation problems, swallowing problems, occurrence of recurrent mouth infections due to poor oral hygiene, or salivary incontinence.

Several reduction techniques have been described (Fig. 6) $[5,6]$. We use the Morgan technique [7], which has been modified by performing a " $\mathrm{W}$ "-shaped ventral incision of the tongue instead of a " $\mathrm{V}$ "-shaped incision to preserve the lingual frenulum. Its preservation ensures better symmetry of 

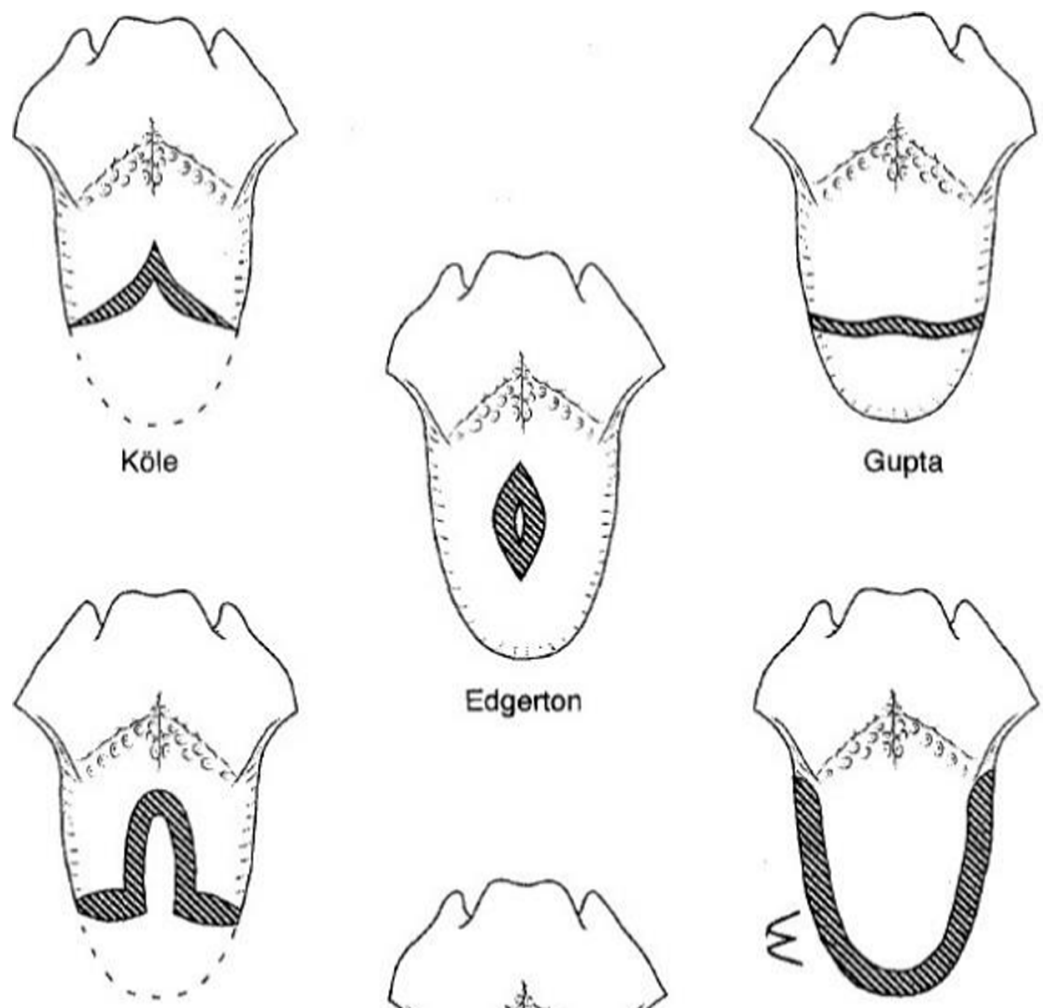

Gupta

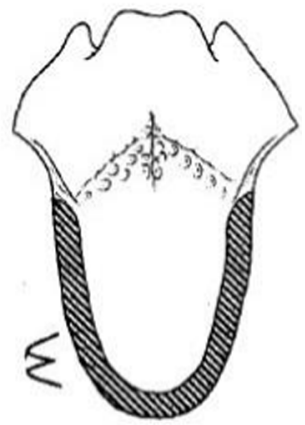

Egyedi \& Obwegeser
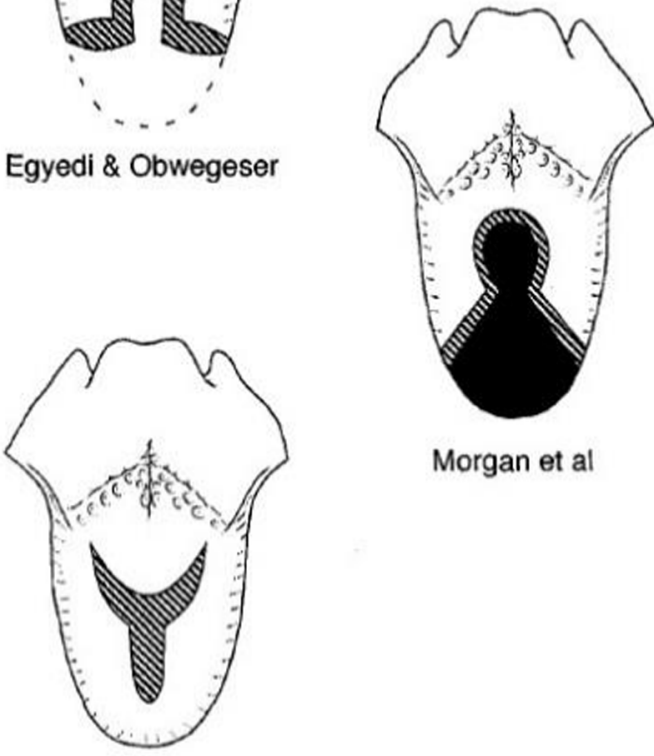

Morgan et al

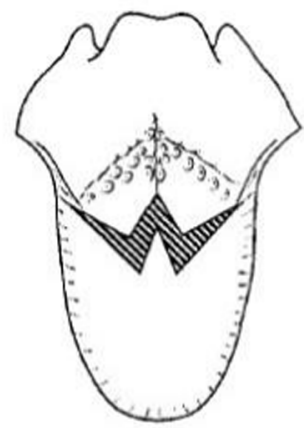

Harada \& Enomoto

Mixter et al

Fig. 6. Diagrams of different reduction glossoplasty techniques.

the tongue, allows better limitation of lingual movements, and thus avoids recurrence because of lingual misalignment. This technique also allows more relevant reduction either in length or in width while preserving the neurovascular bundle, thus preserving gustatory, sensory, and motor functions. Nevertheless, postoperative care of reduction glossoplasty is often complicated by tongue and mouth floor edema, delaying the resumption of normal functions [8].

Rehabilitation consisted essentially of the following [9]: - correction of the tongue position at rest;

- rehabilitation of swallowing with two objectives: (1) breaking the "glossolabial" reflex and (2) achieving efficient back and forth movement of the tongue;
- reading exercises; speech therapy aims to standardize lingual support during the pronunciation of different phonemes: the palatal "L, N, D, T", sibilant " $S$ ", the postalveolar sibilant "CH, J";

- rehabilitation of tongue strength by alternating between a strong contraction of the tongue by tapering the tip "rat tongue" and a sprawl "cat tongue".

This technique was performed in three patients of ages 5 , 8 , and 11 years. We have not found age limit in the literature to perform this surgical technique. At the time of drafting this report, the patients have been followed up for an average of 2 years, with no relapses or complications. 


\section{Conclusion}

Macroglossia is a rare pathology, the source of functional and morphologic complications. Surgical reduction is the treatment of choice. Our technique is simple and minimal, ensuring good functional and morphological results over the long term.

Conflicts of interest: The authors declare that they have no conflicts of interest in relation to this article.

\section{References}

1. Balaji SM. Reduction glossectomy for large tongues. Ann Maxillofac Surg 2013;3:167-172.

2. Jaja Z, Boulaich M, El Ayoubi A, El Ayoubi F, Kettani S, Essakalli S, et al. Syndrome de Beckwith-Wiedemann : évolution favorable des troubles fonctionnels après glossectomie. Med Buccale Chir Buccale 2011; 17:55-57.

3. Raposo A, Preisler G, Salinas F, Muñoz C. Glosoplastía con Técnica de Harada en un Paciente con Síndrome de Down. Int J Odontostomat 2011;5(3):245-248.
4. Maladière $E$, Vacher $C$. Examen clinique en stomatologie. EMC (Elsevier Masson SAS, Paris), Stomatologie 2008;22-010-A-10; Médecine buccale 2008;28-230-P-10.

5. Gasparini G, et al. Surgical management of macroglossia: discussion of 7 cases. Oral Surg Oral Med Oral Pathol Oral Radiol Endod 2002;94:566-571.

6. Davalbhaktaand A, Lamberty BGH. Technique for uniform reduction of macroglossia. Br J Plast Surg 2000;53:294-297.

7. Morgan WE, Friedman EM, Duncan NO, Sulek M. Surgical management of macroglossia in children. Arch Otolaryngol Head Neck Surg 1996;122:326-329.

8. Dutot MC, Soupre V, Vazquez MP, Picard A, Kadlub N. Syndrome de Wiedemann-Beckwith glossectomie de réduction par Ultracision ${ }^{\circledR}$ : une approche novatrice. Rev Stomatol Chir Maxillofac Chir Orale 2015;116(2):82-85.

9. Breton I, Nicolas P, Torres JH. Temps buccal de la déglutition salivaire : physiologie et principes de Rééducation. EMC (Elsevier Masson SAS, Paris), Stomatologie/Odontologie 2000;22-008-A-20; Médecine buccale, 2008;28-165-M-10. 